\title{
Airy pattern approximation of a phased microphone array response to a rotating point source
}

Maarten Debrouwere and David Angland ${ }^{1}$

Faculty of Engineering and the Environment, University of Southampton, Southampton, SO16 7QF, UK

(Dated: 15 February 2017)

Deconvolution of phased microphone array source maps is a commonly applied technique in order to improve the dynamic range and resolution of beamforming. Most deconvolution algorithms require a Point Spread Function (PSF). In this work, it is shown that the conventional definition of the PSF, based on steering vectors, is changed when the source is rotating. The effect of rotation results in an increase in the resolution and aperture of the array. The concept of virtual array positions created by source rotation is used to derive an approximation of the PSF based on an Airy pattern. The Airy pattern approximation is suitable for use in deconvolution of rotating source maps as it is more accurate and is computationally less expensive than the conventional PSF definition. The proposed Airy pattern approximation was tested with both CLEAN and DAMAS deconvolution algorithms. On the same hardware, it was significantly faster when compared to the conventional definition. The limitations of the Airy pattern approximation are shown in a synthesized broadband test case with a high dynamic range. However, in most practical beamforming applications, the proposed Airy pattern approximated PSF for deconvolution is a suitable option considering its accuracy and speed.

Keywords: Phased microphone array; deconvolution; aeroacoustics; beamforming 


\section{INTRODUCTION}

Phased microphone array systems are commonly used in aeroacoustic research ${ }^{1}$. They have been used for source localization on aircraft fly-overs ${ }^{2}$, wind turbines ${ }^{3}$ and wind tunnel models ${ }^{4}$. More recently the technique is being used to analyze the noise generated by contra-rotating open $\operatorname{rotors}^{5-9}$. Conventional beamforming codes are in the frequency domain due to their computational speed advantage. However, the localization of rotating sound sources requires the use of a rotating source beamforming algorithm in the time domain such as ROSI ${ }^{10}$. Other techniques, mostly used for sound source localization on fan blades of turbo-fan engines, include circular harmonics beamforming 11 and virtual rotating microphone imaging $\frac{12}{2}$. However, these techniques apply restrictions on the physical layout of the microphone array. When a conventional beamformer (assuming stationary sources) is applied to rotating sources, the output is a relatively uniform distribution of noise source around the rotation axis since the point source assumption is not valid $\underline{10}$. The output from a rotating source time domain beamformer is a source map that shows the source at the correct location at a particular point in time.

While beamforming has been applied to contra-rotating open rotors as outline above, an example of the potential limitations of using phased microphone arrays to locate sources on a counter rotating open rotor rig is given in Horvath et al. $\underline{\underline{5}}$. In this example, where the array was looking side-on to the rotor rig and the a beamformer assumed compact, stationary and incoherent sources, the source maps produced sources that were not located on the blades. The explanation for this was due to the spiral wavefront pattern propagating away from the open rotor. For most frequencies the spatial location of the source corresponded to the Mach radius, i.e. the radial position at which the lobes of a circumferential spinning mode travel toward the observer at the speed of sound. However, in spite of the example above, beamforming results of rotating sources are still useful to investigate rotor noise, especially in a wind-tunnel or flyover test environment $\underline{13}$.

Deconvolving conventional beamforming source maps is a commonly used technique in phased microphone array processing. Commonly used deconvolution techniques applied to phased microphone array data are CLEAN ${ }^{14}$, DAMAS ${ }^{15}$ and its extensions DAMAS2/3ํㅡㄴ and NNLS17. The wide range of deconvolution techniques used to increase the resolution and dynamic range of conventional beamforming all make use of the array system's response 
to a point source, i.e. the Point Spread Function (PSF). This PSF is generally calculated from the steering vectors of the array $\underline{14}$.

In this work, an approximation for the PSF for rotating sources is proposed to improve the accuracy of existing deconvolution techniques when applied to source maps of rotating sources but also provide a decrease in computational cost. The proposed PSF approximation can be used for any deconvolution method that requires the response of the array to a point source, i.e. the PSF. Firstly, it is demonstrated that the PSF of a rotating source is different to a stationary source. These differences are explained by introducing the concept of a virtual array pattern, created by the rotating motion of the source. Next, an approximation for rotating source PSF's is proposed based on Airy patterns. Ideally one would use the exact PSF for the rotating case but this requires synthesizing data and subsequently processing it with a time domain rotating source beamforming algorithm. This process is computationally expensive since a new exact PSF is needed for each point in the scan plane and for each frequency. The proposed approximation is computationally much less expensive than calculating the exact PSF and for rotating sources is more accurate than the conventional PSF definition based on steering vectors. Experimental results are shown where a comparison is made between deconvolution for rotating sources with the conventional PSF definition and the Airy pattern approximation. Finally, the limitations of the approximation are demonstrated on a test case with synthesized data that is deliberately designed to show where the Airy pattern approximation for the PSF breaks down.

\section{SETUP DESCRIPTION}

In this work. both synthesized and measured data is used. A sketch of the set-up is shown in Fig. 1. The phased microphone system used consists of 56 microphones, which are placed in a multi-arm spiral configuration. The same 56 microphone positions were used for both the synthesized data and in the experiments. The sources are located in the $x-y$ plane at $z=0 \mathrm{~m}$ (the scan plane). The array is positioned at a distance of $0.6 \mathrm{~m}$ from the plane of source rotation. For the initial discussion on the differences between the PSF for a stationary and a rotating source in Section III, a single source is used at various offsets from the rotation axis to demonstrate the effect of source offset on the PSF for a rotating source. In the experiments to demonstrate the deconvolution based on the proposed Airy pattern 
approximation for the PSF in Section V, two sources are used with different frequencies and amplitudes, offset from the rotation axis by a fixed distance of $0.235 \mathrm{~m}$ as shown in Fig. 1.

The experimental rig physically consisted of two small piezo-buzzers mounted on an aluminum bar giving the required separation between the sources. The aluminum bar is fixed to the shaft of a bipolar gear-less stepper motor, for easy rotational speed control. The stepper motor is controlled via an A3967 micro-stepping driver chip and an Arduino Uno board. The whole motor and source assembly is mounted on a wooden support structure to allow free rotation. For the results presented in this article the motor rotates at 100 RPM. One buzzer produces a tone at $2889 \mathrm{~Hz}$ while the other produces a tone at $2917 \mathrm{~Hz}$ and is $2 \mathrm{~dB}$ quieter. The microphones are connected to a pre-amplifier and high-pass filter. Once properly conditioned, each channel is sampled by a National Instruments PXI-4472 card housed in a NI PX-1045 chassis. The sampling frequency is $48 \mathrm{kHz}$ and the total number of samples per channel is $2.048 \times 10^{5}$. The post-processing of the microphone array data is done with a rotating source time domain beamformer, based on the theory presented by Sijtsma ${ }^{10}$. The data sets are divided into 5 equal length blocks to compute an average source map. The FFT block size was $4.1 \times 10^{4}$. This results in a frequency resolution of $1.17 \mathrm{~Hz}$. The field of view $(\mathrm{FOV})$ is a $0.8 \mathrm{~m} \times 0.8 \mathrm{~m}$ area centred at the centre of rotation with a scan field grid spacing of $0.02 \mathrm{~m}$. This results in 1681 grid points.

\section{POINT SPREAD FUNCTIONS FOR ROTATING SOURCE BEAMFORMING}

When performing phased microphone array measurements on rotating sources, such as helicopter blades, wind turbines, open rotors, etc. $\frac{1}{}$, the source motion has a significant effect. Not only does it require a rotating source beamforming algorithm (e.g. ROSI ${ }^{10}$ ), it also modifies the Point Spread Function (PSF) of the system. To demonstrate the effect of source rotation on the response of the array to a point source, data was synthesized for a single source at $3 \mathrm{kHz}$ using the setup in Fig. 1 for both a stationary case and a rotating case. The offset of the rotating source was $0.012 \mathrm{~m}$ from the rotation axis.

The effect of rotation about an axis can be transformed to a frame of reference where the sources are stationary and the array is rotating. This is useful for understanding and explaining the differences between the PSF for rotating and stationary sources. In this frame 


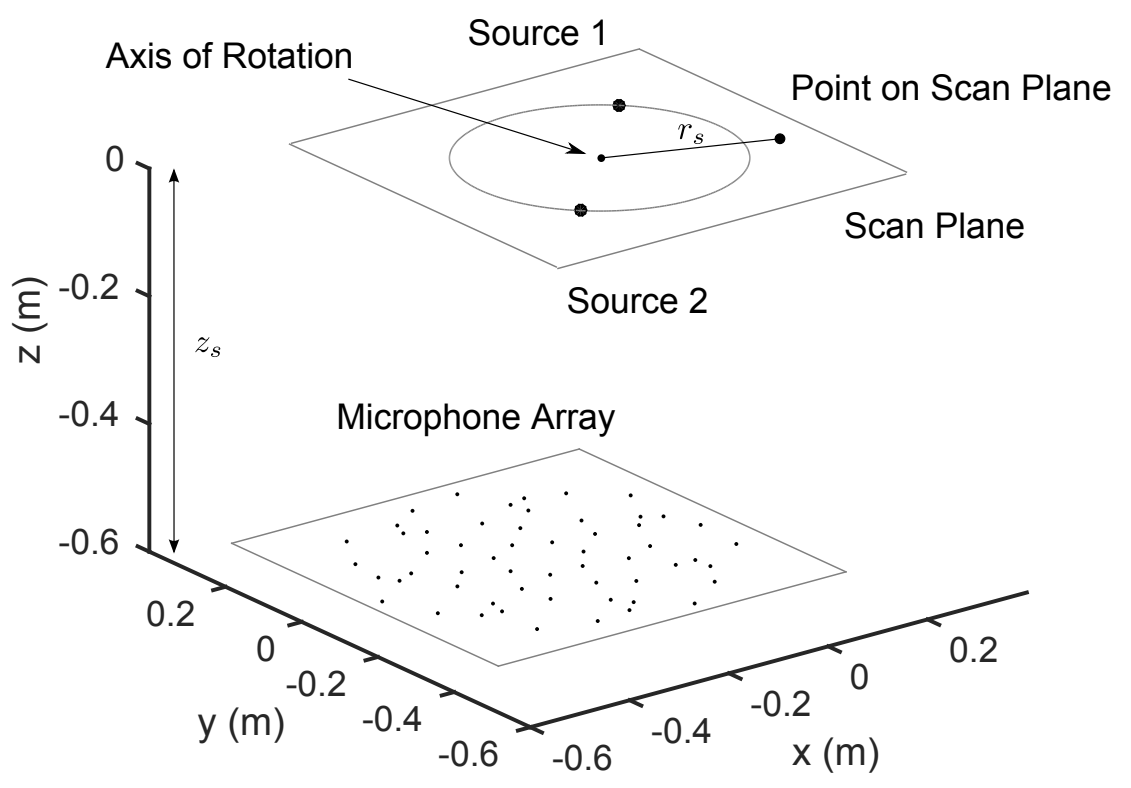

FIG. 1: Sketch of the experimental set-up, showing the microphone array and rotating source locations.

of reference the axis of rotation is located at the origin at the centre of the array is offset by the appropriate amount. This results in a virtual location of microphones due to source rotation. Fig. 2 shows the virtual and initial sensor locations for a simple case rotating at a $100 \mathrm{RPM}$ and a sampling frequency of $48 \mathrm{kHz}$. The offset between the centre of rotation and the centre of the array is $0.12 \mathrm{~m}$ in the $x$ direction.

Figs. 3(a) and 3(b) show the PSF at $3 \mathrm{kHz}$ for a stationary case and rotating case respectively. Fig. 3(a) was computed from the classical PSF definition based on steering vectors and Fig. 3(b) was obtained through simulating a monopole source and subsequently processing that data with a rotating source beamforming algorithm using the setup described above. The rotating PSF's (Fig. 3(b) and later in Fig. (4) has been transposed to the origin for each of the offsets to allow comparison at different offsets.

The most significant difference between the rotating and stationary PSF's in Fig. 3are the side-lobe pattern and levels. The conventional PSF shows discrete side-lobes. The locations and levels of the side-lobes are a function of the array layout. The rotating source PSF shows side-lobes in a concentric pattern around the main peak. Furthermore the power of these concentric side-lobes decays more rapidly compared to the side-lobes for a conventional 


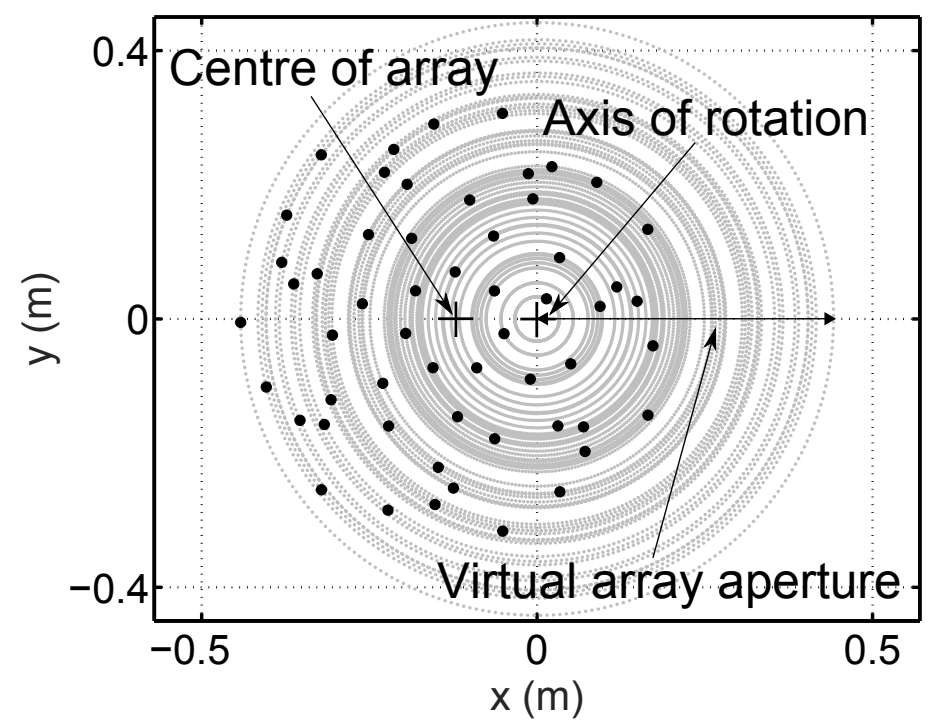

FIG. 2: Virtual (small grey dots) and initial (large black dots) sensor locations for simple rotating case at $100 \mathrm{RPM}$ at a sampling frequency of $48 \mathrm{kHz}$. Every $100^{\text {th }}$ virtual microphone position is shown for clarity. The centre of rotation is about the origin. The centre of the microphone array is located at $x=-0.12 \mathrm{~m}$ and $y=0 \mathrm{~m}$.

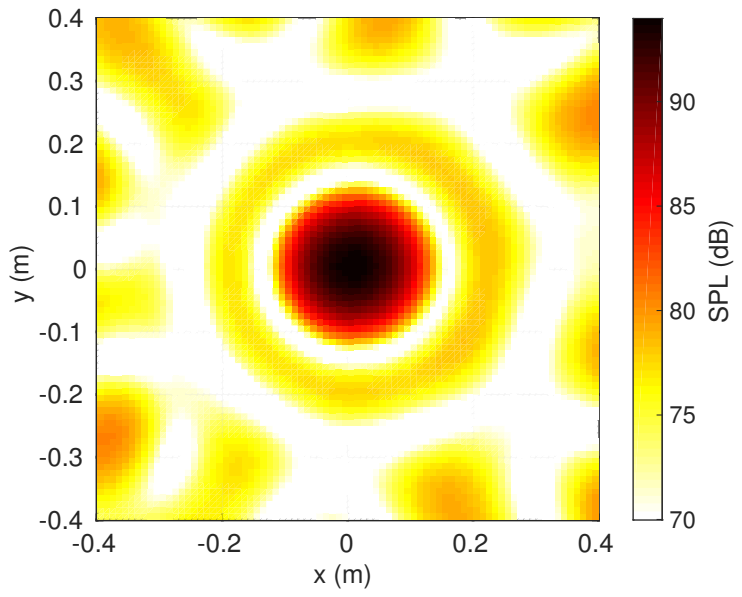

(a)Conventional beamforming applied to a stationary source.

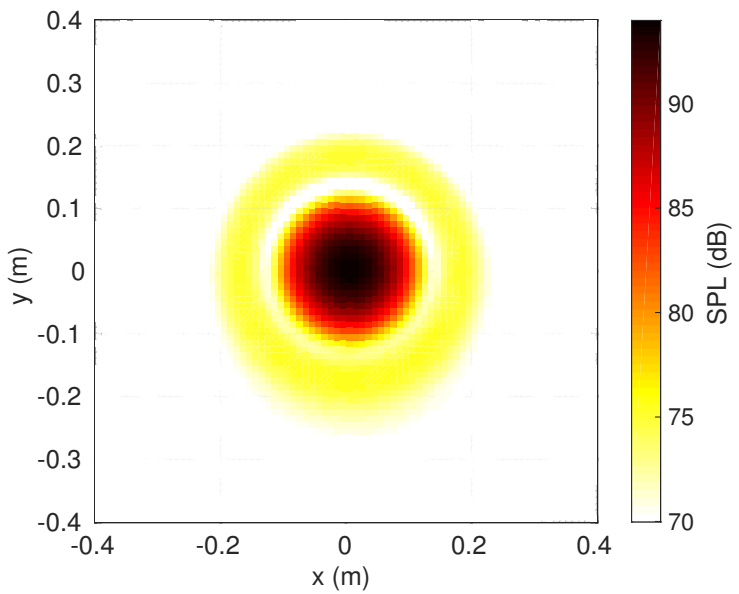

(b)Rotating beamforming applied to a rotating source (source has been transposed to the origin).

FIG. 3: PSF for a $3 \mathrm{kHz}$ monopole source (colour online).

PSF.

These differences are explained using the concept of a virtual array shown in Fig. 2. Since 
the source locations in the scan plane used by the rotating source beamformer move relative to the microphones in the array, the distance and associated time delay between those two points will differ at every sample time. This is in contrast to a stationary measurement where the time delays between a certain scan point and microphone remain constant. Changing the frame of reference, from one where the microphone positions are fixed in the array and the sources rotate around a common axis, to a frame of reference where the source locations are fixed and the microphones or sensors rotate, yields a map of the virtual microphone positions for every instance of time (Fig. 2). In this frame of reference, the axis of rotation is coincident with the $z$-axis, which makes the virtual microphone positions $(x, y)$ defined by Equations 1 and 2, where $x^{\prime}$ and $y^{\prime}$ are the original microphone positions, $\Omega$ the rotational velocity of the system $t_{s}$ are the discrete sample times of the system.

$$
\begin{aligned}
& x=x^{\prime} \cos \left(\Omega t_{s}\right)-y^{\prime} \sin \left(\Omega t_{s}\right) \\
& y=x^{\prime} \sin \left(\Omega t_{s}\right)+y^{\prime} \cos \left(\Omega t_{s}\right)
\end{aligned}
$$

The virtual array consist of many more sensor points compared to the case without rotation. This leads to a more densely populated sensor disk. This is the reason why the PSF in a rotating case has less significant side lobes as shown in Fig. 3. Secondly, if the microphone array is not centred around the centre of rotation (which is the case in Fig. 3(b)), the main lobe will become more narrow and thus effectively increases the resolution of the phased microphone array. Another interesting aspect of the virtual array pattern is the aperture. Because the centre of the physical microphone array is not aligned with the centre of rotation, the aperture of the virtual array (indicate by a dashed black arrow in Fig. 2) is larger compared to the aperture of the original array pattern. This causes the main lobe in the PSF to be narrower and therefore increases the resolution. These effects are demonstrated using the synthesized data in Fig. 3.

An experiment where a rotating buzzer is measured with a phased microphone array system corresponding to the pattern shown in Fig. 2 empirically confirmed these observations. In Fig. 4 radial sections through the main peak of the source maps from three different set-ups are shown. The first case is a stationary measurement with no rotation which serves as a reference. In the second case the buzzer is rotating at 100 RPM and there is an off-set of $0.12 \mathrm{~m}$ between the centre of rotation and the centre of the array. The virtual array 
pattern associated with this set-up is shown in Fig. 2, The increased aperture does lead to a narrower main lobe compared to the stationary case. Due to the more densely packed sensors disk due to rotation, the side-lobe levels are also reduced.

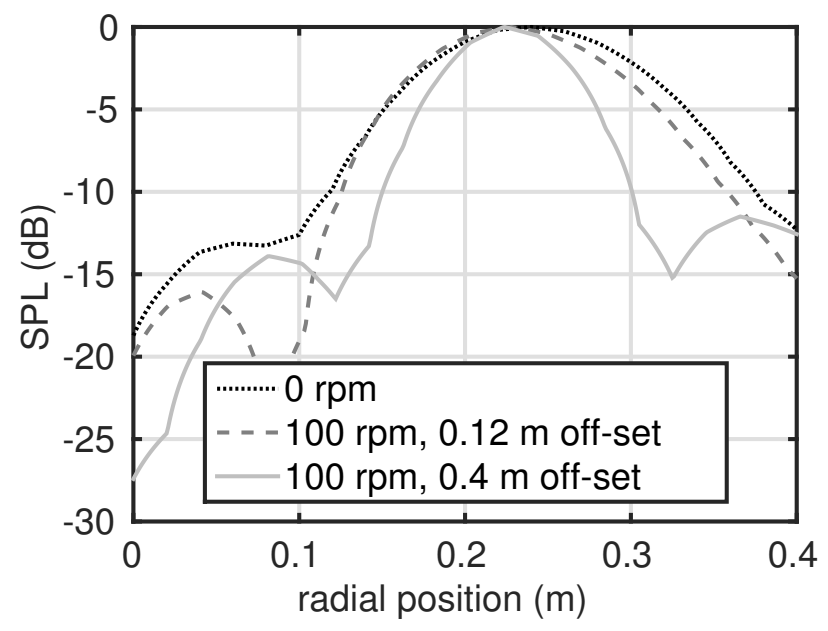

FIG. 4: Section through the main peak in source map of a rotating buzzer for a stationary case and two rotating cases with different off-sets.

If the off-set between the array centre and centre of rotation is increased to $0.4 \mathrm{~m}$, the virtual aperture is increased even more. However, in this case the sensor disk has a lower sensor density as the microphones distribution is more sparse away from the centre of the array as shown in Fig. 2. As expected this leads to an increase in resolution but leads to an increase in the side-lobe levels compared to the small off-set case. This demonstrates experimentally the two effects of aperture and resolution with a rotating source compared to the corresponding stationary case.

Since many deconvolution methods require the PSF, the use of a stationary PSF based on steering vectors is not correct due to the differences between the stationary and rotating PSF as demonstrated here. It is possible to use the exact PSF for a rotating case but previously explained in the Introduction, this is a computationally expensive procedure. Therefore, an approximation of the PSF for rotating sources based on Airy patterns is proposed. 


\section{APPROXIMATION OF PSF FOR ROTATING SOURCE BEAMFORMING}

From optics it is known that the PSF related to a perfect circular aperture is given by an Airy pattern (Born and Wolf표 $)$. The same principle can be applied to an acoustic system. Since the virtual array pattern created by having a rotating source approximates a fully covered sensors disk, the corresponding PSF can therefore be approximated by an Airy pattern. With typical multi-arm spiral array designs, the amount of sensors near the centre of the virtual array tends to be low (Fig. 2), hence a better approximation for the PSF is found using the expression for an obscured Airy pattern (Born and Wolf ${ }^{18}$ ) given below,

$$
I(\theta)=\frac{2 I_{0}}{\left(1-\epsilon^{2}\right)^{2}}\left(\frac{J_{1}(\gamma)}{\gamma}-\frac{\epsilon J_{1}(\epsilon \gamma)}{\gamma}\right)^{2},
$$

where,

$$
\gamma=k R_{\max } \sin (\theta)
$$

In Equation 3, $J_{1}$ is the Bessel function of order one and of the first kind and $k=2 \pi / \lambda$ is the wave number. $R_{\max }$ and $\epsilon$ are properties of the virtual array pattern representing the maximum radius of the virtual array after rotation and aperture obstruction ratio respectively.

The angle $\theta$ is the angle between two vectors. The first is the vector from the origin of the array to each point in the scan plane calculated for every scan point $(\mathbf{A})$. The second is the vector pointing towards the PSF main lobe position from the array origin $(\mathbf{B})$. In the CLEAN algorithm for example, this is done each iteration as part of the algorithm to search for the peak location in the source map $\stackrel{14}{\underline{4}}$. This results in a single vector for each iteration that points from the origin of the array towards the peak in the beamforming map. The angle $\theta$ is then calculated at every scan point using the definition of the dot product. The angle is,

$$
\theta=\cos ^{-1}\left(\frac{\mathbf{A} \cdot \mathbf{B}}{\|\mathbf{A}\|\|\mathbf{B}\|}\right)
$$

All the points in the scan plan will be at an angle with respect to the array normal vector. The incident waves also have curvature due to the spherical propagation of the sound field so the incident angle of incoming waves must be accounted for. The effective aperture radius accounts for the propagation direction of the incident waves $\frac{19}{}$. For a point on the scan plane 
located at a radial distance of $r_{s}$ from the origin and for a horizontal distance between the scan plane and the array of $z_{s}$ (Fig. 1), the nearfield effective aperture radius is given by (Allen et al..$^{19}$ ),

$$
R_{\max _{\mathrm{eff}}}=r \sin \left[\frac{\frac{\pi}{2}-\tan ^{-1}(a)-\tan ^{-1}(b)}{2}\right]
$$

where

$$
\begin{aligned}
& r=\sqrt{r_{s}^{2}+z_{s}^{2}}, \\
& a=\frac{z_{s}}{r_{s}+R_{\max }}, \\
& b=\frac{r_{s}-R_{\max }}{z_{s}} .
\end{aligned}
$$

$R_{\max }$ is the maximum radius of the rotating virtual array. The minimum effective radius can also be calculated by replacing $R_{\max }$ with $R_{\min }$ in Equation 6 ,

The aperture obstruction ratio is defined as the effective radius of the obscured part of the sensor disk divided by the maximum effective radius,

$$
\epsilon=\frac{R_{\text {min }_{\text {eff }}}}{R_{\text {max }_{\mathrm{eff}}}} .
$$

This accounts for the fact that the whole array disk is not filled with microphones, particular in the centre of the array.

To match the beamforming output, the intensity pattern is converted to pressure squared values with Equation 8 and common reference values $I_{\text {ref }}=1 \times 10^{-12} \mathrm{~W} / \mathrm{m}^{2}$ and $p_{\text {ref }}=$ $2 \times 10^{-5} \mathrm{~Pa}$.

$$
p^{2}=I \frac{p_{\mathrm{ref}}^{2}}{I_{\mathrm{ref}}}
$$

Fig. [5]shows an approximation of a PSF at $3 \mathrm{kHz}$ using the Airy pattern method described in Section II. The approximate PSF in Fig. 5 corresponds to the same setup used for the PSF's given in Fig. 3(b). The Airy pattern PSF in Fig. 5 matches the exact rotating PSF better compared to the conventional PSF, both shown previously in Fig. 3, The Airy pattern PSF displays a small increase in resolution caused by the larger virtual aperture. Also the side-lobe patterns of the actual rotating PSF and the Airy pattern approximation resemble each other very well. In both cases only one side lobe ring is visible around the main lobe and further away from the main lobe all levels have decayed $24 \mathrm{~dB}$ below the peak value. 


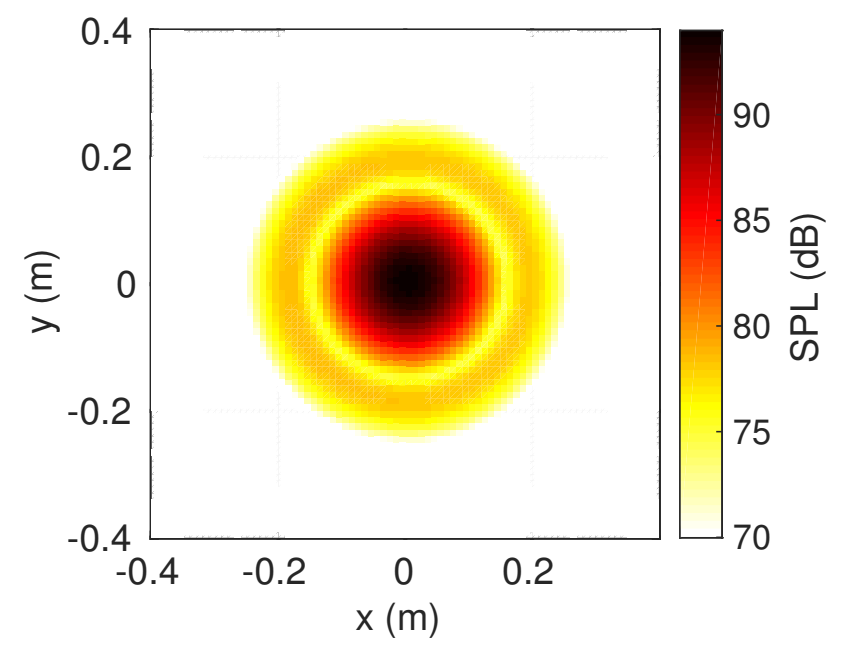

FIG. 5: Approximation of PSF at $3 \mathrm{kHz}$ by Airy pattern (colour online).

\section{DECONVOLUTION WITH AIRY PATTERN PSF}

Because an Airy pattern is a better representation of the PSF of a phased microphone system when used to measure rotating sources, it is proposed to use this approximation in deconvolution techniques to improve their performance. As mentioned previously, ideally one would use the exact PSF for a rotating case, as shown in Fig. 3(b), but this requires synthesizing data and subsequently processing it with a time domain rotating source beamforming algorithm. This process is computationally expensive since a new PSF is needed for each point in the scan plane and for each frequency. The Airy pattern approximation is suitable for use in deconvolution techniques as not only is it accurate, it is very quick to compute. Indeed it is even quicker to compute than the conventional PSF method based on steering vectors.

In order to evaluate the performance of deconvolution techniques using the Airy pattern PSF definition for a practical beamforming example, a simple experimental case is used. Two rotating buzzers are measured with a phased microphone array after which the source map is deconvolved with both the CLEAN 14 and DAMAS 15 methods. The experimental data was processed using both the conventional PSF definition based on steering vectors and the Airy pattern PSF approximation. The experimental setup was shown in Fig. 1.

All source maps included in this section are plotted on a $24 \mathrm{~dB}$ scale and the path of the two buzzers is indicted with a grey dashed line. For reference the original source map calculated by the rotating source time domain beamformer is depicted in Fig. 6. The position 
of the two sources are correctly located by the rotating source beamforming algorithm along the path of rotation. The source map also captures the small power difference between the two buzzers. It should be noted that even on a $24 \mathrm{~dB}$ scale very few side lobes or spurious sources are visible. For the case without source rotation with an identical set-up, the maximum side-lobe levels are approximately $9 \mathrm{~dB}$ below the peak value. In the rotating case the highest side lobe level is approximately $16 \mathrm{~dB}$ below the peak level. As discussed previously in Section IV, this reduction in side-lobe levels is due to the much denser sensor disk of the virtual array.

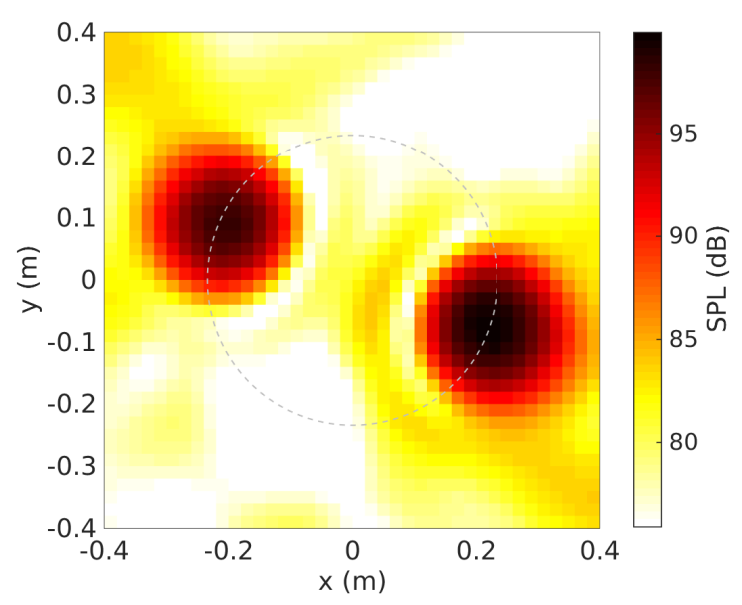

FIG. 6: Source map from time domain rotating source beamforming at 2889 and $2917 \mathrm{~Hz}$ (colour online).

The CLEAN algorithm $\frac{14}{}$ was modified to work with a time domain rotating beamformer code and with the Airy pattern approximation for the PSF. For a given frequency, the maximum and minimum effective radius of the rotating virtual array are calculated. From these the aperture obscurity factor can be calculated. As part of the CLEAN algorithm, the location and amplitude of the peak in the original beamforming map are determined. Once the location of the peak is known, for each point in the scan plane the angle $\theta$ is calculated. The appropriately scaled PSF using the Airy pattern approximation (Equation 3) is determined and this is subtracted from the original map. This procedure is repeated iteratively.

Fig. 7(a) shows the final source map obtained after deconvolution with the CLEAN algorithm using the conventional PSF definition based on steering vectors. Although most of the power in the source map is located around the two buzzer positions, some lower 
intensity spurious sources are visible. The deconvolved source map calculated with the PSF approximation based on Airy patterns, shown in Fig. 7(b), on the other hand concentrates almost all of the energy at the positions of the the buzzers. Comparing the total energy contained within the two source regions (defined as a 3 by 3 pixel region) to the total energy in the source maps gives an indication of how well the deconvolution algorithm performs. If it performed perfect, all the energy would be found in the two peak regions and this ratio would be 1 . However, when the conventional PSF definition is used this energy ratio is found to be 0.95 and with the Airy pattern approximation the energy ratio becomes 0.99 .

As well as improving the accuracy compared to the conventional definition the PSF applied to a rotating source, the Airy pattern PSF method also provides a significant speedup. On the same hardware the CLEAN algorithm using the Airy pattern PSF's was more than 35 times faster compared to the conventional method. This is partly due to faster convergence because of the better match between the approximated and actual PSF, but it is mainly due to the fact that calculating an Airy pattern, defined by Equation 3 , is much faster than the steering vector counterpart.

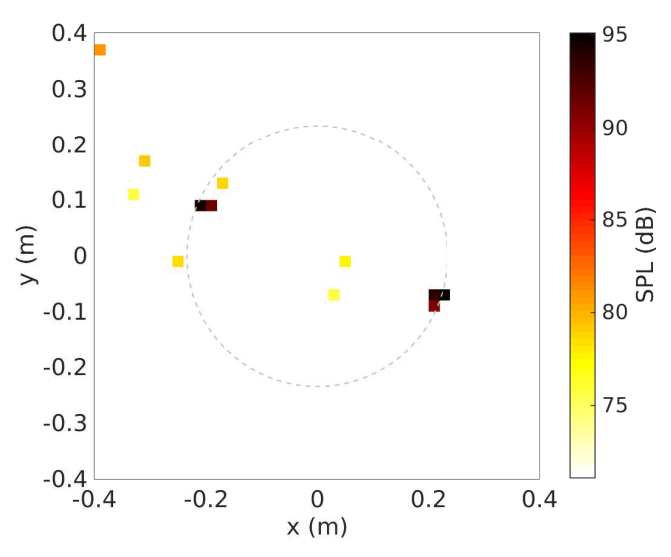

(a)Conventional PSF definition.

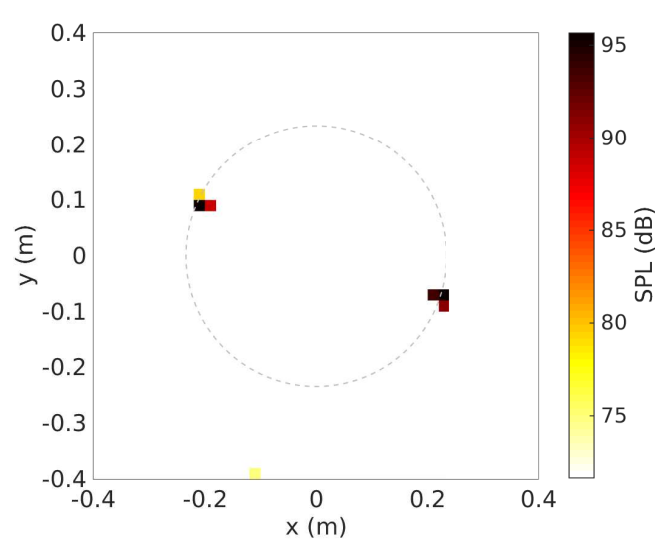

(b)Airy pattern PSF definition.

FIG. 7: Source maps after CLEAN deconvolution (colour online).

Deconvolving Fig. 6 with the DAMAS method yields results that are consistent with the source maps from CLEAN deconvolution. Figs. $8(a)$ and $8(b)$ show the final source maps after DAMAS deconvolution for both the conventional and Airy pattern PSF definitions respectively. Similar to the previous results, the source map obtained after deconvolution with the conventional PSF definition contain more and higher intensity spurious sources 
compared to the deconvolved map using the Airy pattern PSF's. The ratio of source energy and total energy for the conventional DAMAS method is 0.91 , while the DAMAS method with the Airy pattern PSF's increases this ratio to 0.94 .

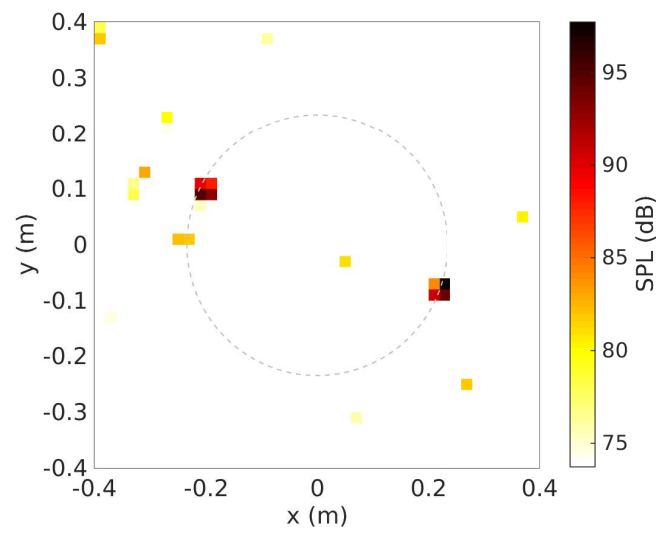

(a)Conventional PSF definition.

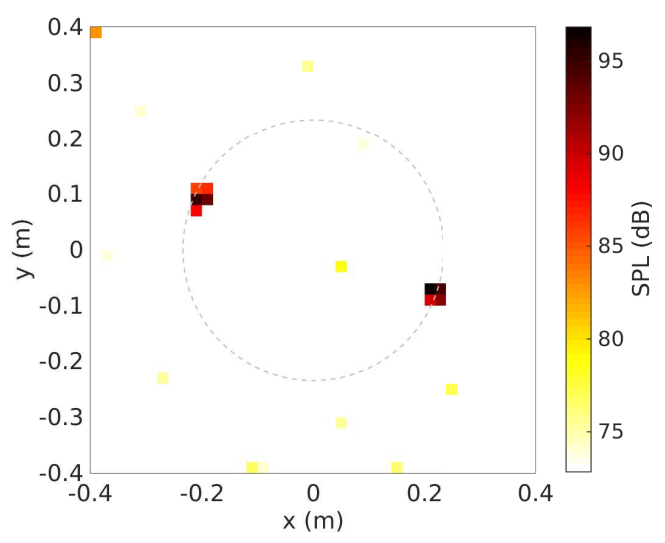

(b)Airy pattern PSF definition.

FIG. 8: Source maps after DAMAS deconvolution (colour online).

More relevant for DAMAS deconvolution is the speed-up achieved due to the new PSF definition. It has previously been reported in literature that DAMAS requires relatively large computational resources when applied to realistic cases $\frac{16,20}{}$. In this case, both methods required a similar number of iterations in order to converge, the DAMAS algorithm using the Airy pattern PSF approximations was still 14 times faster when compared to the conventional method. This method can be applied in cases with large fields of view or grid points, where the shift invariant PSF methods such as DAMAS2 or DAMAS3 ${ }^{16}$ are not applicable due to the higher differences in angles between the array and scan points in the domain.

\section{LIMITATIONS OF AIRY PATTERN APPROXIMATION}

As demonstrated in Section V, deconvolution of rotating source beamforming maps with the approximated Airy pattern PSF performs better compared to the conventional PSF definition both in terms of accuracy (fewer spurious noise sources) and an increase in computational speed. This was demonstrated on an experimental case with a realistic dynamic range. However the Airy Pattern PSF is still an approximation and there are some limits to its use. These limitations originate from the errors made in approximating the exact PSF. 
Even though the Airy pattern approximation matches the exact PSF much closer than the conventional definition, there are two main sources of error which need to be considered.

Fig. 9 shows both an exact one-dimensional PSF calculated from synthetic data and an Airy pattern approximation. The main lobe and first sidelobes are approximated very well. The first discrepancy occurs at the valley point next to the main lobe (at $x= \pm 0.13$ m). As $\gamma$ approaches 3.8317 in Equation 3, the Bessel function goes to zero and the PSF power therefore goes to minus infinity $\mathrm{dB}$. This is repeated for every valley point in the PSF approximation. The exact PSF does not show the same trend. For the example shown in Fig. 9 the difference in the first valley point is $11 \mathrm{~dB}$ and for the second valley point the error increases to almost $60 \mathrm{~dB}$.

The second main difference between the exact and approximated PSF occurs at the higher order side lobes (further away from the main lobe). In contrast to the first side lobe, there are big differences in power levels between these higher order side lobes. For this case, the difference between the second side lobe for the exact and Airy pattern PSF is approximately $9 \mathrm{~dB}$.

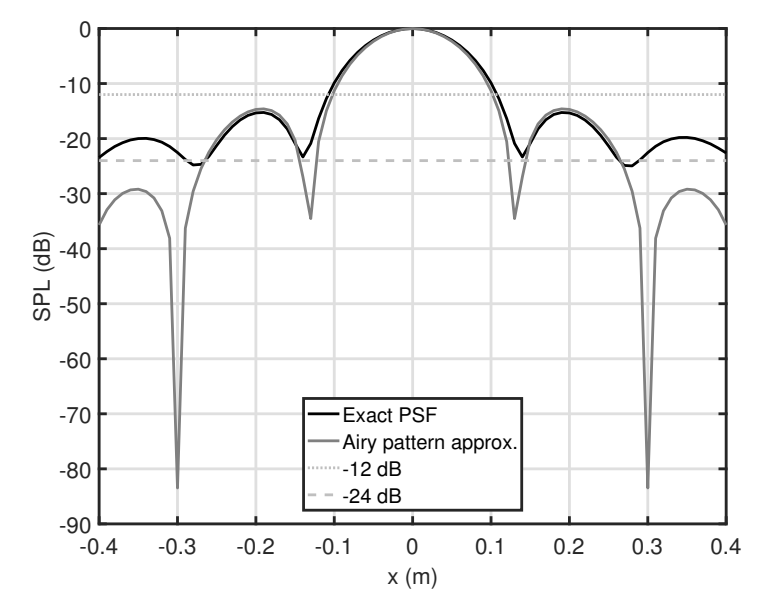

FIG. 9: Difference between exact PSF and Airy pattern approximated PSF.

Before analysing the impact of these discrepancies between the two PSF's further, it is important to note that even though some of the approximation errors are very high (up to $60 \mathrm{~dB})$, their relative importance compared to the main lobe and first side-lobe is small. Since in a rotating source phased microphone array measurement, the side lobe levels decay quickly (Section III), they are less likely to appear in the final source map. In the synthesized data shown in Fig. 9 and the experimental data shown in Fig. 6, none of the side lobes are 
within $12 \mathrm{~dB}$ of the peak value. Only when using a dynamic range of $24 \mathrm{~dB}$ are the side lobes visible. The same holds true for the minima in the PSF's. Since these are the local minima they inherently have a low power level. Therefore, the impact of the errors in the approximation of the Airy pattern on a practical beamforming application is limited since it is common to use a dynamic range between 12 and $24 \mathrm{~dB}$ depending on the precision of the measurement equipment and environment.

Nevertheless these differences from the exact PSF may affect the final deconvolved map as a consequence of underestimating the higher order side lobe levels and minima. When the approximate Airy pattern PSF value is lower than the actual PSF, not all of the side lobe power will be removed after a deconvolution iteration. The remaining side lobe power might be interpreted as an independent source in a subsequent iteration. High precision cases with a large dynamic range are more prone to these errors in the approximation because there are only significant differences between the actual and the approximated Airy PSF $16 \mathrm{~dB}$ below the peak level.

To demonstrate the previously mentioned limitations, a challenging high frequency and high dynamic range test case is synthesized. Four white noise sources are simulated on the corners of a $0.2 \mathrm{~m}$ by $0.2 \mathrm{~m}$ square, rotating at $100 \mathrm{RPM}$. Source 1 is located in the bottom right corner and subsequent sources follow in clockwise fashion. The source strength is different for each location with a maximum difference of $18 \mathrm{~dB}$, which will challenge the dynamic range limitations of the Airy PSF approximation. The same array configuration and data processing parameters were chosen as for the experimental case, except for the scan plane. The field of view is reduced to a $0.4 \mathrm{~m}$ by $0.4 \mathrm{~m}$ square but the grid point spacing is also reduced to $0.005 \mathrm{~m}$, giving a total of 6561 grid points.

Fig. 10 shows the source map at $10 \mathrm{kHz}$ produced by the rotating source time domain beamforming algorithm. The evaluation frequency of $10 \mathrm{kHz}$ is deliberately chosen to make sure several high order side-lobe rings and multiple valleys are included in the field of view. The side lobe rings associated with Source 1 are clearly visible in the source map given in Fig. 10.

The deconvolved source maps shown in Fig. 11(a) for the CLEAN algorithm and in Fig. 11(b) for DAMAS algorithm reveal the impact of approximating the PSF by an Airy pattern. As expected many low power (around $16 \mathrm{~dB}$ below the peak value) spurious sources are added to the deconvolved map to compensate for the discrepancies between the exact 


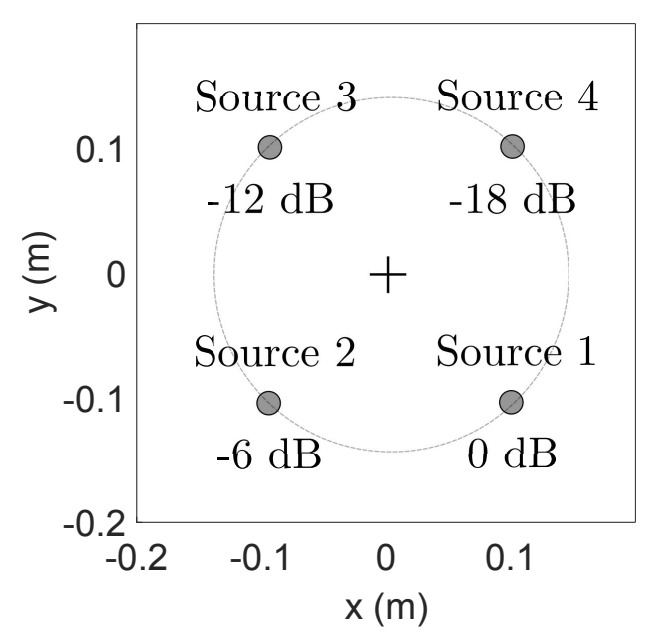

(a)Setup of problem. Source strengths are shown relative to Source 1 . The cross in the centre of the image shows the centre of rotation.

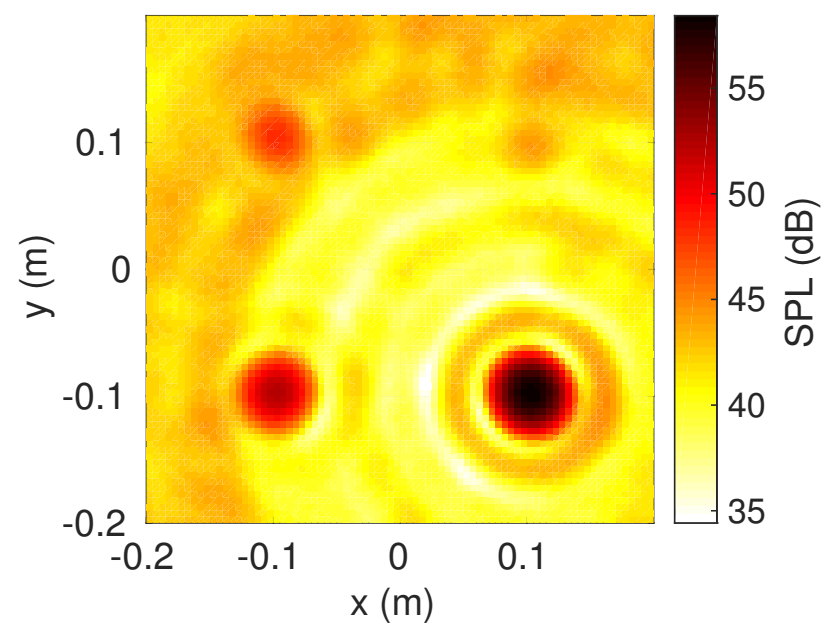

(b)Time domain rotating source beamforming result at $10 \mathrm{kHz}$.

FIG. 10: White noise, high frequency, high dynamic range test case rotating at $100 \mathrm{RPM}$ (colour online).

and approximated PSF. These spurious sources form concentric patterns around the actual sources due to the errors in the valley and higher order side-lobe regions, which also form concentric circles. In both cases a lot of the energy is still located around the source locations with a source to total energy ratio for the CLEAN method of 0.58 and 0.44 for DAMAS, but the spurious sources pollute the overall result. The integrated source power for the four different source regions relative to Source 1 are tabulated in table I. Apart from Source 2 , both deconvolution methods deviate from the correct values due to the approximations made in the Airy pattern PSF.

When examining these source maps it is important to note that this test case was deliberately chosen to demonstrate the limitations of the approximations made in the Airy pattern PSF. When limiting the dynamic range to $16 \mathrm{~dB}$ or selecting a lower frequency or using harmonic sources instead of white noise sources, the Airy pattern approximation matches the exact PSF sufficiently closely such that the deconvolved maps do not contain any spurious sources. 


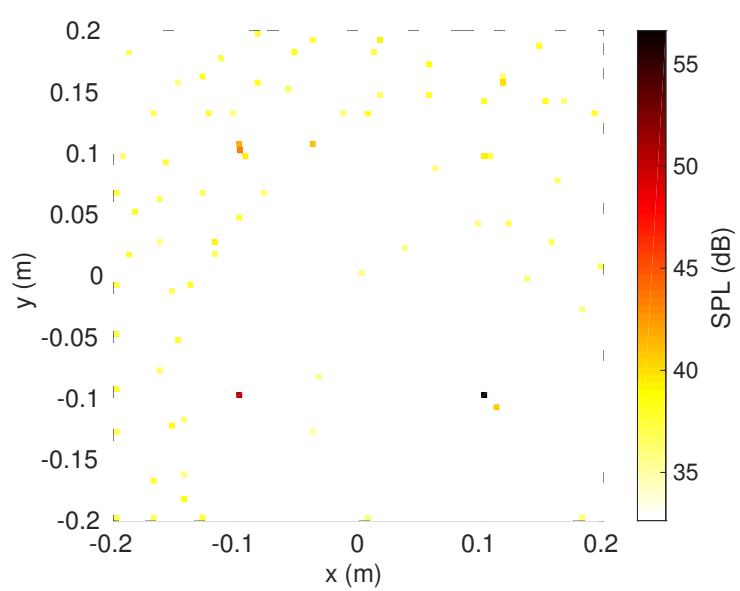

(a)CLEAN.

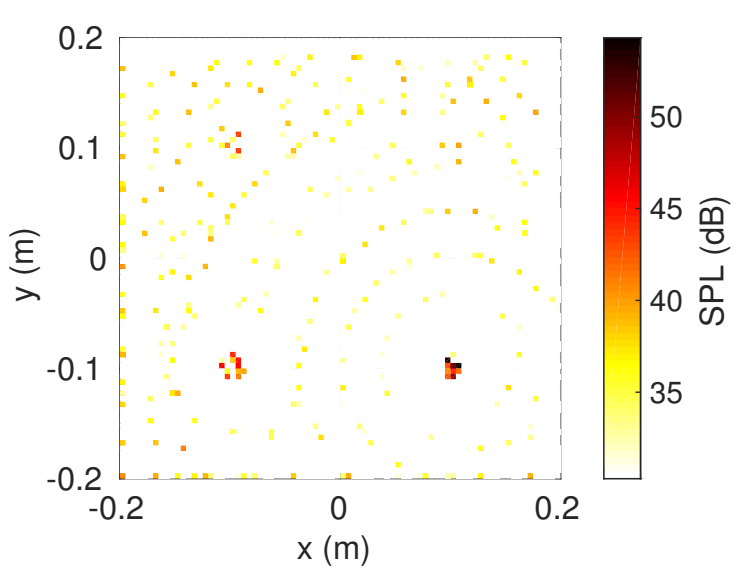

(b)DAMAS.

FIG. 11: Deconvolved source map at $10 \mathrm{kHz}$ with Airy pattern PSF approximation (colour online).

\section{DECONVOLUTION WITH A SHIFT INVARIANT ROTATIONAL PSF}

For the case where the Airy pattern approximation is not valid, and the domain of interest is smaller than the (virtual) array diameter, a shift invariant exact PSF can be used. For rotating sources this exact PSF is still different from the conventional PSF definition used for example in the DAMAS2 technique. Instead of quickly obtaining the PSF through the steering vectors, it requires the simulation of a point source which is subsequently processed with the rotating source time domain beamformer. An example of this was already shown in Fig. 3(b). Since this is a computationally intensive procedure, it is computationally expensive to recalculate a PSF for every point in the scan plane. Therefore, the shift invariant assumption is made and the PSF is simply translated to each grid position. This method does however require the simulation and calculation of a new PSF for every change in set-up which can be inconvenient, especially during an experimental campaign. The shift invariant assumption will introduce a new error but if the field of view remains small enough this error will not be significant.

Both CLEAN and DAMAS deconvolution methods are applied with the shift invariant PSF to the source map given by Fig. 10. In both resulting deconvolved maps, Fig. 12(a) and 12 (b), fewer spurious sources are detected. In the case of the CLEAN algorithm almost 
all energy is contained within the source regions with a source to total energy ratio of 0.99. Although the deconvolved DAMAS map features more spurious sources, with a source to total energy ratio of 0.95 , it better estimates the integrated relative power levels given in table I. The DAMAS algorithm combined with the shift invariant PSF has a maximum difference of only $0.2 \mathrm{~dB}$ when compared to the expected power level values.

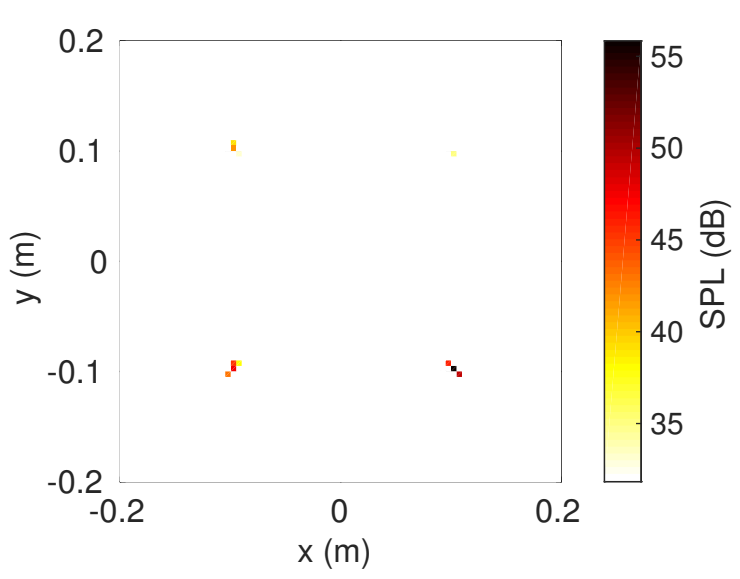

(a)CLEAN.

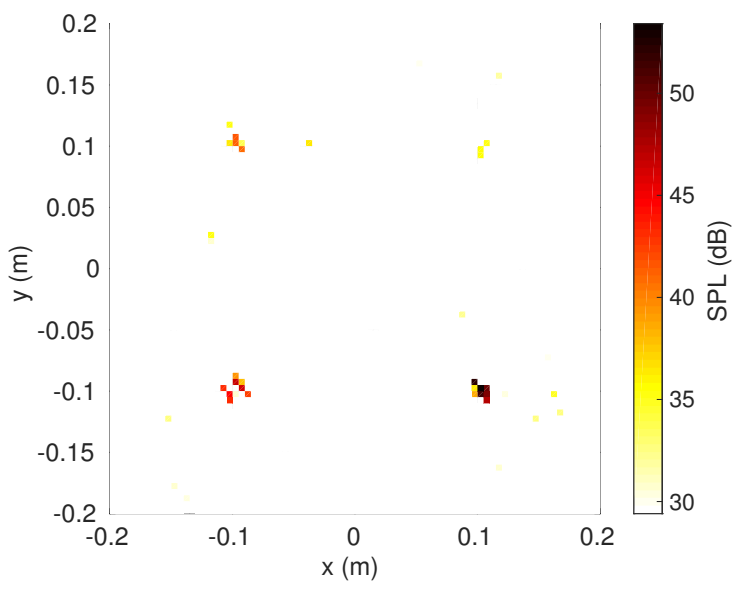

(b)DAMAS.

FIG. 12: Deconvolved source map at $10 \mathrm{kHz}$ with shift invariant PSF (colour online). 


\section{CONCLUSIONS}

The conventional Point Spread Function (PSF) definition based on steering vectors introduces an error in the case of rotating source beamforming. Differences between a stationary and a rotating source PSF were shown from both synthetic and experimental data. The side lobes for rotating sources form a concentric pattern around the main lobe compared to the discrete side lobes present in a conventional PSF. There were two additional effects of rotation. Firstly, the side lobe levels in the rotating case were found to decay faster compared to the stationary case. Only the first side lobe (closest to the main lobe) was within $24 \mathrm{~dB}$ of the peak value. Secondly, a decrease in main lobe width was found in both the experimental and numerical cases for the rotating source case. These difference were explained by introducing the concept of a virtual array pattern created by the motion of the source. This virtual array pattern has a very dense sensor disk and an increased aperture leading to the reduction in side-lobe levels and increase in resolution.

The rotating source PSF was approximated by an obscured Airy pattern derived from the properties of the virtual array pattern. The approximation agreed closely with the exact PSF for the main lobe and first side lobe. The Airy pattern approximation also captured the change in main lobe width as well as the change in side lobe pattern and levels. Because higher order side lobe decay rapidly for rotating sources, the approximation is well suited for deconvolution of rotating source beamforming maps.

The Airy pattern approximation for the PSF was applied to both the CLEAN and DAMAS deconvolution algorithms. The test cases showed an improvement in both accuracy and computational performance when using the Airy pattern approximated PSF compared to the conventional PSF definition based on steering vectors. For practical cases, with a realistic dynamic range, where higher order side lobes can assumed to be of less importance, deconvolution with the Airy pattern PSF's is suggested as a better alternative to using the conventional PSF definition. It is more accurate for rotating cases, resulting in less spurious noise sources and is computationally less expensive compared to the conventional definition of the PSF. On the same hardware the CLEAN algorithm using the Airy pattern PSF's was more than 35 times faster compared to the conventional method. This is partly due to faster convergence because of the better match between the approximated and actual PSF, but it is mainly due to the fact that calculating an Airy pattern is much faster than the steering 
vector counterpart. In this case, both methods required a similar number of iterations in order to converge, the DAMAS algorithm using the Airy pattern PSF approximations was still 14 times faster when compared to the conventional method.

The limitations of the Airy pattern approximation were outlined by synthesizing a test case with a high dynamic range and broadband frequency content. This case was deliberately chosen to demonstrate where the Airy pattern approximation breaks down. This resulted in multiple high order side-lobes in the original source map. The errors made by the approximation, especially in the minima and higher order side-lobe peaks, were not negligible in this case used to test the limits of the approximation. The deconvolved maps were polluted by spurious sources compensating for differences between the approximated and actual PSF. These compensating sources formed a concentric pattern coinciding with the minima and higher order side lobe pattern. The use of the exact but shift invariant PSF was suggested as an alternative for cases requiring a high dynamic range. Since the field of view was limited, the shift invariant assumption was valid and the deconvolved source maps no longer featured the spurious compensating sources.

Even though the exact shift invariant PSF technique worked well, it is important to note that the domain restrictions required for the shift invariant assumption to be correct and the need to calculate the exact PSF means it is potentially inconvenient for practical applications. In most practical beamforming applications of rotating sources, with a typical dynamic range, using an Airy pattern approximated PSF for rotating source deconvolution is a suitable option considering its convenience and accuracy.

\section{REFERENCES}

${ }^{1} \mathrm{U}$. Michel, "History of Acoustic Beamforming," in Proceedings on CD of the 1st Berlin Beamforming Conference, Berlin, BeBeC-2006-01, (2006).

${ }^{2}$ P. Sijtsma, "Phased Array Beamforming Applied to Wind Tunnel And Fly-Over Tests," SAE Technical Paper 2010-36-0514, (2010).

${ }^{3}$ R. C. Ramachandran, H. Patel and G. Raman, "Localization of Wind Turbine Noise Sources Using a Compact Microphone Array with Advanced Beamforming Algorithms," in Proceedings on CD of the 4th Berlin Beamforming Conference, Berlin, BeBeC-2012-25, (2012). 
${ }^{4}$ P. A. Ravetta, R. A. Burdisso, and W. F. Ng, "Phased Array Technologies Development at Virginia Tech: Application to Landing Gear Noise Source Identification," ENIEF 2004 Congress on Numerical Methods and its Applications, San Carlos de Bariloche, Argentina, 8-11 November, (2004).

${ }^{5}$ C. Horváth, E. Envia, and G. G. Podboy, "Limitations of Phased Array Beamforming in Open Rotor Noise Source Imaging," AIAA Journal, 52:8, 1810-1817, (2014).

${ }^{6}$ S. Funke, L. Kim and H. A. Siller, "Microphone-Array Measurements of a Model Scale Contra-Rotating Open Rotor in a Reverberant Open Wind-Tunnel," in 17th AIAA/CEAS Aeroacoustics Conference, Portland, OR, AIAA Paper 2011-2766, (2011).

${ }^{7}$ S. Funke, L. Kim, H. Siller, "Acoustic Measurements of a Contra-Rotating Open Rotor in an Open Jet Wind-Tunnel," Int. J. of Aeroacoustics, 11:2, 197-212, (2012).

${ }^{8}$ V. Fleury and A. Chélius, "Analysis of Contra Rotating Open Rotors Flyover Noise Data by Beamforming Techniques," in Proceedings of Meetings on Acoustics, 19, 030123 (2013). ${ }^{9}$ P. Chiariotti, M. Martarelli, E. P. Tomasini and P. Castellini, "Aeroacoustic Source Localization on Open Rotor Aircraft Model in Wind Tunnel Tests," in 43rd Int. Congress on Noise Control Engineering, Melbourne, (2014).

${ }^{10}$ P. Sijtsma, S, Oerlemans and H. Holthusen, "Location of Rotating Sources by Phased Array Measurements," in 7th AIAA/CEAS Aeroacoustics Conference, Maastricht, AIAA Paper 2001-2167, (2001).

${ }^{11}$ P. Sijtsma, "Circular Harmonics Beamforming with Multiple Rings of Microphones," in 18th AIAA/CEAS Aeroacoustics Conference, Colorado Springs, CO, AIAA Paper 20122224, (2012).

${ }^{12}$ P. R. Dougherty and B. E. Walker, "Virtual Rotating Microphone Imaging of Broadband Fan Noise," in 15th AIAA/CEAS Aeroacoustics Conference, Miami, FL, AIAA Paper 2009-3121, (2009).

${ }^{13} \mathrm{C}$. Horvath, "Beamforming Investigation of Dominant Counter-Rotating Open Rotor Tonal and Broadband Noise Sources," AIAA Journal, 53:6, 1602-1611 (2015)

${ }^{14}$ P. Sijtsma, "CLEAN Based on Spatial Source Coherence," Int. J. of Aeroacoustics, 6:4, 309-333, (2007).

${ }^{15}$ T. F. Brooks and W. M. Humphreys, "A Deconvolution Approach for the Mapping of Acoustic Sources (DAMAS) Determined from Phased Microphone Arrays," J. of Sound and Vibration, 294:4-5, 856-879, (2006). 
${ }^{16}$ P. R. Dougherty, "Extensions of DAMAS and Benefits and Limitations of Deconvolution in Beamforming," in 11th AIAA/CEAS Aeroacoustics Conference, Monterey, CA, AIAA Paper 2005-2961, (2005).

${ }^{17}$ C. L. Lawson and R. J. Hanson, Solving Least Squares Problems, (Soc. for Industrial and Appl. Mathematics, Philadelphia ,1974), pp. 160-165.

${ }^{18}$ M. Born and E. Wolf, Principles of Optics: Electromagnetic Theory of Propagation, Interference and Diffraction of Light, 7th edition, (Cambridge University Press, Cambridge, 1999), pp. 395-416.

${ }^{19}$ C. S. Allen, W. K. Blake, R.P. Dougherty, D. Lynch, P. T. Soderman and J. R. Underbrink, Aeroacoustic Measurements, edited by T. J. Mueller, (Springer-Verlag, Berlin, Heidelberg, 2002), pp. 112.

${ }^{20}$ T. Yardibi, J. Li, P. Stoica and L. N. Cattafesta, "Sparsity Constrained Deconvolution Approaches for Acoustic Source Mapping," The J. Acoust. Soc. of Am., 123, 2631-2642 (2008) 


\section{TABLES}

TABLE I: Integrated source power from deconvolved maps relative to Source 1. All values in $\mathrm{dB}$.

\begin{tabular}{|c||c|c||c|c||c|}
\hline \multicolumn{1}{|c||}{} & \multicolumn{2}{c||}{ Airy pattern PSF } & \multicolumn{2}{c||}{ Shift Invariant PSF } & \\
\hline Source & CLEAN & DAMAS & CLEAN & DAMAS & Exact \\
\hline 1 & 0 & 0 & 0 & 0 & 0 \\
2 & -6.3 & -5.8 & -6.5 & -6.2 & -6 \\
3 & -10.2 & -10.5 & -13 & -12.1 & -12 \\
4 & -15.4 & -14.8 & -21.9 & -18 & -18 \\
\hline
\end{tabular}

